\title{
Stereotactic radiosurgery boost to the resection bed for oligometastatic brain disease: challenging the tradition of adjuvant whole-brain radiotherapy
}

\author{
Brian J. Karlovits, D.O., ${ }^{1}$ Matthew R. Quigley, M.D., ${ }^{2,3}$ \\ Stephen M. Karlovits, M.D., ${ }^{1,3}$ Lindsay Miller, ${ }^{1}$ Mark Johnson, M.S., 1 \\ Olivier Gayou, Ph.D., ${ }^{1}$ and Russell Fuhrer, M.D. ${ }^{1,3}$ \\ Departments of ${ }^{I}$ Radiation Oncology and ${ }^{2}$ Neurosurgery, Allegheny General Hospital, Pittsburgh; and \\ ${ }^{3}$ Drexel University College of Medicine, Allegheny Campus, Philadelphia, Pennsylvania
}

\begin{abstract}
Object. Whole-brain radiation therapy (WBRT) has been the traditional approach to minimize the risk of intracranial recurrence following resection of brain metastases, despite its potential for late neurotoxicity. In 2007, the authors demonstrated an equivalent local recurrence rate to WBRT by using stereotactic radiosurgery (SRS) to the operative bed, sparing $72 \%$ of their patients WBRT. They now update their initial experience with additional patients and more mature follow-up.

Methods. The authors performed a retrospective review of all cases involving patients with limited intracranial metastatic disease ( $\leq 4$ lesions) treated at their institution with SRS to the operative bed following resection. No patient had prior cranial radiation and WBRT was used only for salvage.

Results. From November 2000 to June 2009, 52 patients with a median age of 61 years met inclusion criteria. A single metastasis was resected in each patient. Thirty-four of the patients each had 1 lesion, 13 had 2 lesions, 3 had 3 lesions, and 2 had 4 lesions. A median dose of $1500 \mathrm{cGy}$ (range 800-1800 cGy) was delivered to the resection bed targeting a median volume of $3.85 \mathrm{~cm}^{3}$ (range $0.08-22 \mathrm{~cm}^{3}$ ). With a median follow-up of 13 months, the median survival was 15.0 months. Four patients $(7.7 \%)$ had a local recurrence within the surgical site. Twenty-three patients (44\%) ultimately developed distant brain recurrences at a median of 16 months postresection, and $16(30.7 \%)$ received salvage WBRT ( 8 for diffuse disease [ $>3$ lesions], 4 for local recurrence, and 4 for diffuse progression following salvage SRS). The median time to WBRT administration postresection was 8.7 months (range $2-43$ months). On univariate analysis, patient factors of a solitary tumor (19.0 vs 12 months, $\mathrm{p}=0.02)$, a recursive partitioning analysis (RPA) Class I (21 vs 13 months, $\mathrm{p}=0.03)$, and no extracranial disease on presentation ( 22 vs 13 months, $\mathrm{p}=0.01$ ) were significantly associated with longer survival. Cox multivariate analysis showed a significant association with longer survival for the patient factors of no extracranial disease on presentation $(\mathrm{p}=0.01)$ and solitary intracranial metastasis $(\mathrm{p}=0.02)$. Among patients with no extracranial disease, a solitary intracranial metastasis conferred significant additional survival advantage (43 vs 10.5 months, $\mathrm{p}=0.05$, log-rank test). No factor (age, RPA class, tumor size or histological type, disease burden, extent of resection, or SRS dose or volume) was related to the need for salvage WBRT.

Conclusions. Adjuvant SRS to the metastatic intracranial operative bed results in a local recurrence rate equivalent to adjuvant WBRT. In combination with SRS for unresected lesions and routine imaging surveillance, this approach achieves robust overall survival (median 15 months) while sparing $70 \%$ of the patients WBRT and its potential acute and chronic toxicity. (DOI: 10.3171/2009.9.FOCUSO9191)
\end{abstract}

Key Words

\section{radiosurgery $\bullet$}

\section{oligometastasis}

$\mathrm{T}$ RADITIONALLY, WBRT has been the standard approach to minimize the risk of intracranial recurrence following resection of brain metastases. Almost 2 decades ago, Patchell et al. ${ }^{16}$ established the superiority of resection of solitary metastases followed by WBRT compared with WBRT alone with regard to survival, local control, and length of functional independence. A subsequent study by the same group failed

Abbreviations used in this paper: $\mathrm{MLC}=$ multileaf collimator; MMSE = Mini-Mental State Examination; RPA = recursive partitioning analysis; RTOG = Radiation Therapy Oncology Group; SRS = stereotactic radiosurgery; SRT = stereotactic radiotherapy; $\mathrm{WBRT}=$ whole-body radiation therapy. to demonstrate a survival advantage for the addition of WBRT compared with resection alone in patients with a solitary intracranial metastasis, although the likelihood of local and distant recurrence and death from neurological causes were significantly reduced by WBRT. ${ }^{15}$ Due to potential delayed neurocognitive effects associated with WBRT, investigators have evaluated the use of partial brain irradiation in the form of SRS in lieu of WBRT after resection of brain metastases. ${ }^{4,13}$ They showed that despite WBRT's superior control of brain recurrence in sites other than the resection bed, SRS after resection resulted in equivalent survival and neurological preservation. ${ }^{1,21}$ At Allegheny General Hospital in 2000, we ad- 
opted a combined approach to the treatment of patients with 1-4 brain metastases utilizing resection of solitary and bulky metastases followed by SRS to the operative bed and remaining lesions.

In 2007, we published our initial experience of 32 patients treated with this novel approach. ${ }^{17}$ We demonstrated a local recurrence rate of $6.25 \%$, which was equivalent to the recurrence rate achieved with WBRT. The median survival was 16.4 months, and $72 \%$ of our patients were spared WBRT. Since this publication, authors from other institutions have published similar focused techniques with favorable outcomes. ${ }^{5,8,22}$

This paper updates our experience using this treatment approach with additional patients and more mature follow-up.

\section{Methods}

From November 2000 to June 2009, 52 patients with newly diagnosed limited intracranial metastatic disease ( $\leq 4$ lesions) were offered resection of solitary accessible lesions or bulky $(>3.5 \mathrm{~cm})$ disease in the setting of multiple lesions, followed by adjuvant SRS. No patient had received prior intracranial radiotherapy. The SRS was delivered to the operative bed and remaining lesions 4-6 weeks after resection. The patient records were retrospectively reviewed, and data regarding the following variables were recorded: age; sex; primary pathology; RTOG RPA class; ${ }^{6}$ extent of extracranial disease (based on preoperative staging enhanced CT or PET/CT of chest, abdomen, and pelvis); the number, site, and size of brain metastases; extent of resection (gross vs subtotal, as evidenced by 24-hour postoperative MR imaging); postoperative complications and hospitalization; and SRS dose and treatment volume. This study was approved by the Allegheny General Hospital Institutional Review Board as a retrospective review of existing clinical records.

Patients underwent resection assisted by frameless neuronavigation techniques. All patients underwent a gadolinium-enhanced brain MR imaging study (slice thickness 2-3 mm) 4-6 weeks postoperatively. This was followed on the same day by placement of a stereotactic head frame under local anesthesia. A treatment planning CT of the head was then obtained and the MR imaging and CT image sets were fused. The contrast-enhancing edges of the operative bed and other lesion(s), if any, were delineated by the radiation oncologist and neurosurgeon on the MR images. Treatment plans for patients treated before May 2008 (44 patients) were designed for optimum conformality using one of the 3 following techniques on the XKnife RT-3.0.1 software (Radionics): 1) circular arcs using cones, 2) collimated circular arcs using cones and jaws, or 3) conformal fixed beams using the Radionics 4-mm-leaf mini-MLC. They were treated on a Varian 2100C linear accelerator with a couch mount for fixation of the head ring. Treatment plans for patients treated after May 2008 (8 patients) were designed using one of the 2 following techniques on the iPlan software (BrainLAB): 1) circular arcs using cones, or 2 ) conformal arcs using the 5-mm-leaf MLC (Siemens Medical Solutions). They were treated on a Siemens Primus or Siemens Artiste linear accelerator. Early on, dose selection was variable,

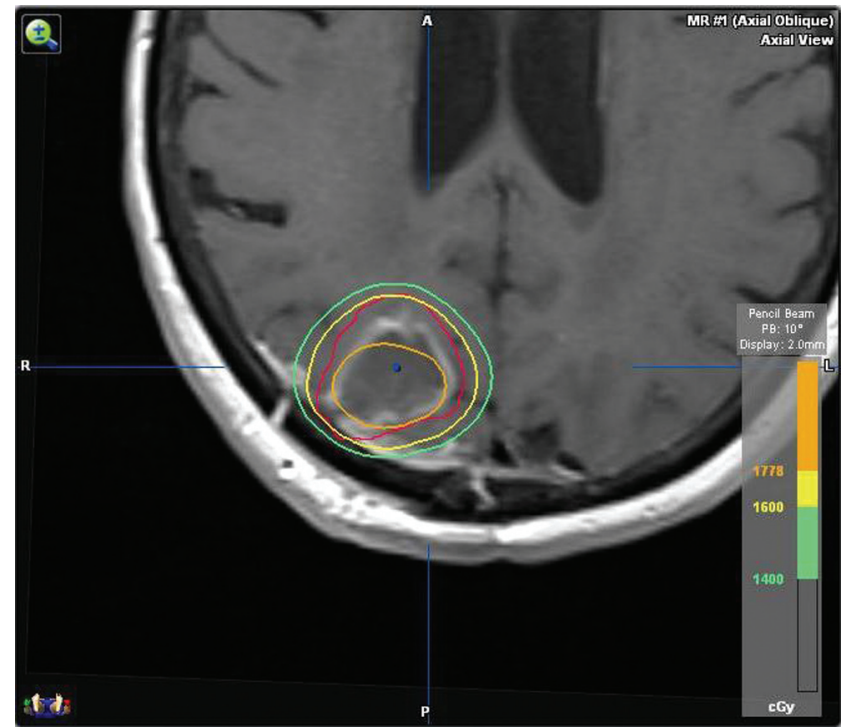

FIG. 1. Axial dose distribution of a typical treatment plan showing planning treatment volume (red line) encompassing the enhanced resection cavity on T1-weighted MR images.

physician dependent, and based upon RTOG 90-05 volume-dependent SRS guidelines. ${ }^{20}$ Target volumes, operative beds, and other lesion(s) were also prescribed to the 80-90\% isodose line without a margin. In our current approach, planning treatment volumes encompass the tumor bed/lesion plus T1-weighted MR imaging enhancement plus a 2-mm margin, and dose is prescribed to the $80 \%$ isodose line (Fig. 1). The prescribed dose is volume dependent, based on RTOG guidelines, and ranges from 15 to 20 Gy.

Follow-up MR imaging was performed 1 month post-SRS and then at 3-month intervals thereafter. If fewer than 3 new lesions were diagnosed on follow-up imaging and if they were $<3.5 \mathrm{~cm}$ in size and patients were classified as RTOG RPA Class 1 or 2, the treatment was salvage SRS to the new lesions; otherwise WBRT was recommended.

Survival and postoperative time of recurrence were determined by the method of Kaplan and Meier ${ }^{9}$ and statistical calculations performed with MedCalc for PC 9.2.0.1. Local brain recurrences were defined as the presence of nodular contrast enhancement adjacent to the resection cavity on MR images. Distant brain recurrences were defined as the presence of limited ( $\leq 3$ lesions) or diffuse metastases outside of the SRS resection cavity volume.

\section{Results}

Fifty-two consecutive patients were treated in the above manner. There were 24 women, and the median age of the entire cohort was 61 years (range 31-85 years). The primary tumor type was non-small cell lung in 24 , 9 breast, 3 each of renal and colon, 2 gastric, 5 unknown, and 1 each from 6 various other sites. Twenty-two and 30 patients were classified as RTOG RPA Class 1 and Class 2, respectively. Each patient had a single metastasis removed; 34 patients had a solitary metastasis, 13 har- 


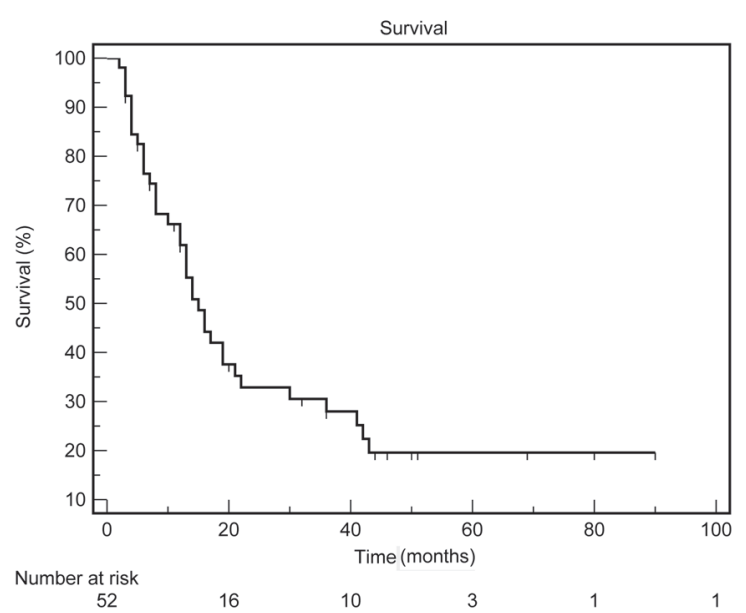

FIG. 2. Kaplan-Meier survival plot of the study population.

bored 2 lesions, 3 patients had 3 lesions, and 2 had 4 . The median size of the resected tumor size was $2.7 \mathrm{~cm}$ (range $2-5 \mathrm{~cm}$ ), and 17 of the resected tumors were located in the posterior fossa. All but 4 patients had complete resections as judged by 24-hour postoperative MR imaging. Operative complications included 2 new visual field cuts that were anticipated due to tumor location and 1 case of culture-negative meningitis. The median duration of postoperative hospitalization was 3 days.

Patients returned for SRS a median of 41 days after resection. The postoperative bed was treated with a median dose of $1500 \mathrm{cGy}$ (range 800-1800 cGy) to the 80$90 \%$ isodose line, with a median target volume of 3.85 $\mathrm{cm}^{3}$ (range $0.08-22 \mathrm{~cm}^{3}$ ). A single isocenter was used. The 25 remaining lesions received a median dose of 1650 cGy (range 700-2000 cGy), with a median target volume of $1.32 \mathrm{~cm}^{3}$ (range $0.06-9.2 \mathrm{~cm}^{3}$ ).

The median survival for the current cohort of $52 \mathrm{pa}-$ tients was 15.0 months (Fig. 2) with a 13-month median follow-up. The updated median survival of the original cohort of 32 patients was 16.5 months with a 17 -month median follow-up. At the time of the analysis, 15 patients

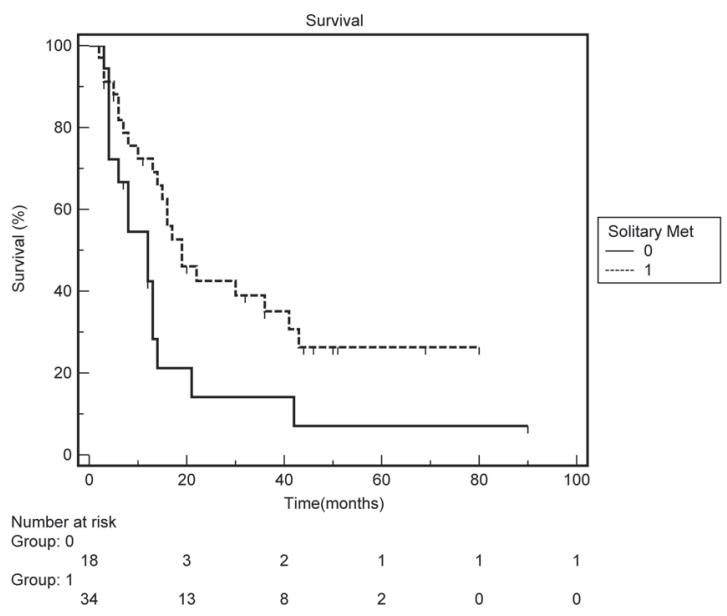

FIG. 3. Kaplan-Meier survival plot of the study population stratified by whether the intracranial deposits were solitary (1) or not (0).

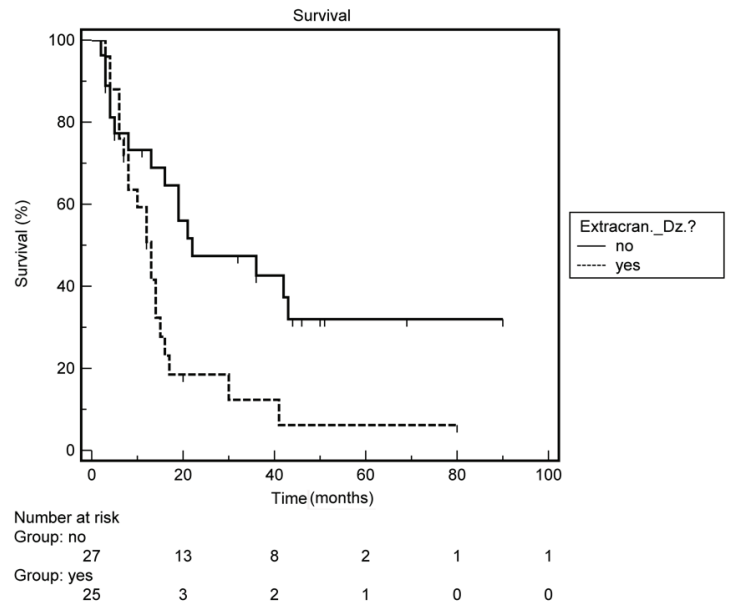

FIG. 4. Kaplan-Meier survival plot of the study population stratified by presence or absence of extracranial disease at presentation.

were still alive. Univariate log-rank analysis showed the following factors to be significantly associated with longer survival: solitary tumor (19.0 vs 12 months, $p=0.02$ ) (Fig. 3), RPA Class 1 (21 vs 13 months, $\mathrm{p}=0.03$ ), and no extracranial disease (22 vs 13 months, $\mathrm{p}=0.01$ ) (Fig. 4). Cox multivariate analysis showed significant associations with longer survival for only 2 factors: no extracranial disease $(p=0.01)$ and solitary metastasis $(p=0.02)$. Among patients with no extracranial disease, a solitary metastasis conferred a significant additional survival advantage (43 vs 10.5 months, $p=0.05$, log-rank test) (Fig. 5).

There were 4 local recurrences within the surgical site (7.7\% of 52 cases), although there was no statistically significant relationship between dose or volume and local control $(\mathrm{p}=0.46$, logistic regression). The median time to local recurrence after resection was not reached. A total of 23 patients (44\%) ultimately developed distant brain recurrences at a median of 16 months postresection. Sixteen (30.7\%) received salvage WBRT, 4 for ini-

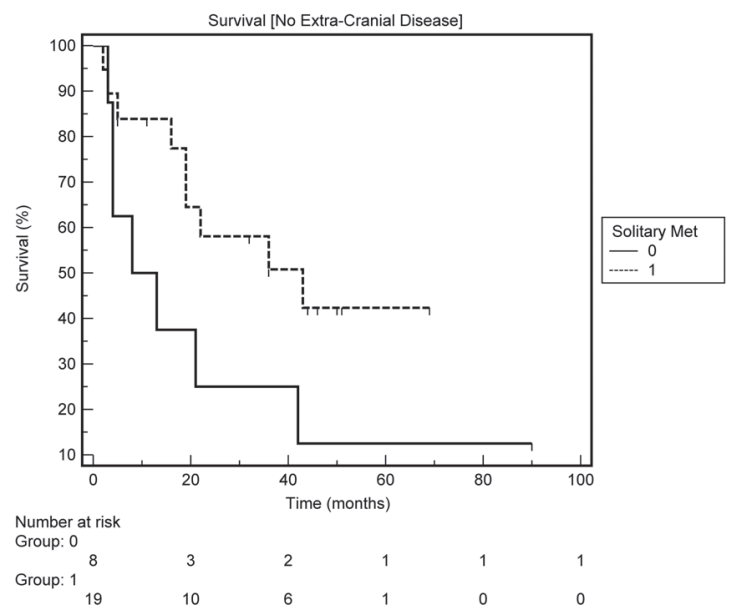

FIG. 5. Kaplan-Meier survival plot of only those patients with no extracranial disease, stratified by whether the intracranial disease was solitary (1) or not (0). 
B. J. Karlovits et al.

TABLE 1: Studies reporting results of postresection SRS*

\begin{tabular}{|c|c|c|c|c|c|c|c|}
\hline Authors \& Year & $\begin{array}{l}\text { No. of } \\
\text { Pts }\end{array}$ & $\begin{array}{l}\text { Median } \\
\text { FU (mos) }\end{array}$ & SRS Technique & Dose in Gy & $\begin{array}{c}\text { Local Tumor } \\
\text { Bed Control } \\
(\%)\end{array}$ & $\begin{array}{l}\text { Median Overall } \\
\text { Survival (mos) }\end{array}$ & $\begin{array}{c}\text { Salvage } \\
\text { WBRT } \\
\text { Rate (\%) }\end{array}$ \\
\hline Kresl et al., 2003 & 61 & 18.3 & Gamma Knife & 16 (median) & 70 & 14.9 & 23 \\
\hline Soltys et al., 2008 & 72 & 8.1 & CyberKnife & 18.6 (median) & 86 & 15.1 & 19 \\
\hline Do et al., 2009 & 30 & NA & LINAC & $\begin{array}{r}\text { SRS 15-18; SRT } \\
22-27 \text { (ranges) }\end{array}$ & 88 & 12 & 47 \\
\hline Jagannathan et al., 2009 & $47 \dagger$ & 10 & Gamma Knife & 19 (mean) & 94 & $10 \%$ & 21 \\
\hline present study & 52 & 13 & LINAC & 15 (median) & 93 & 15 & 30 \\
\hline
\end{tabular}

* FU = follow-up; LINAC = linear accelerator; NA = not available; Pts = patients.

$\dagger$ Three patients received upfront adjuvant WBRT.

* Reported as median duration until death.

tial local and then diffuse recurrence and 12 for diffuse disease ( 4 of these 12 having been previously treated with salvage SRS). Three received salvage SRS alone for limited disease, and their survival was comparable to that of the entire group ( $p=0.81, \log$-rank test). Four patients with advanced extracranial disease died at a median of 1 month (range 1-3 months) after recurrence without further treatment. The median time to WBRT administration postresection was 8.7 months (range 2-43 months). No factor examined (age, RPA class, size or histology of the tumor, disease burden, extent of resection, or SRS dose or volume) predicted the need for subsequent WBRT. No patient experienced greater than Grade 1 toxicity from the SRS treatment.

\section{Discussion}

Adjuvant WBRT is an accepted therapy following resection in patients with limited intracranial metastatic disease and controlled extracranial disease. Although WBRT has been shown to decrease local and distant brain recurrence and death from neurological causes, a survival advantage has not been demonstrated when WBRT was added to resection or SRS in randomized trials. ${ }^{1,15}$ Wholebrain radiation therapy has also been associated with both acute detriment in quality of life and delayed neurocognitive effects. ${ }^{3,4,10,13}$ We therefore adopted and published a novel approach of offering patients with 1-4 brain metastases resection of solitary and bulky intracranial metastatic disease followed by SRS to the operative bed and remaining lesions. ${ }^{17}$

The rationale for adjuvant SRS to the operative bed is to maintain the local control benefits of radiation and surgery without introducing the risks of acute and chronic morbidities associated with WBRT. Our observed local recurrence rate of $7.7 \%$ compares favorably with published local failure rates at the surgical site following WBRT (range 10-20\%) and is superior to the $46 \%$ failure rate reported for resection alone. ${ }^{15,16}$ Utilizing a similar adjuvant SRS approach to the operative bed, Soltys et al. ${ }^{22}$ reported an overall local failure rate of $14 \%$ in 69 irradi- ated beds with $100 \%$ control achieved by adding a 2-mm margin around their resection cavities. Do et al. ${ }^{5}$ demonstrated a $12 \%$ local failure rate when using either adjuvant SRS or SRT to 33 resection cavities. Jagannathan et al. ${ }^{8}$ recently reported a $6 \%$ local recurrence rate in 47 patients who received adjuvant SRS, including 3 who received upfront WBRT. ${ }^{8}$

Distant brain recurrence occurred in $44 \%$ of our patients - a rate similar to the recurrence rates of $37-53 \%$ reported in previous studies in which WBRT was not used. ${ }^{7,15,18,22}$ Although higher than the $28 \%$ in our original report, the rate of $44 \%$ is more in keeping with what has been reported in other literature and the increase is probably the result of more mature follow-up. ${ }^{17}$ Concern regarding distant brain recurrences when omitting WBRT is a source of controversy. Patchell et al. ${ }^{15}$ and Regine et al. ${ }^{18}$ reported increased death from neurological causes when omitting WBRT following resection and "decreased neurologic deficit progression-free survival" with SRS alone, respectively. However, Aoyama et al., ${ }^{1}$ in a Phase III randomized trial comparing the combination of SRS and upfront WBRT to SRS alone, failed to demonstrate a significant difference in deaths from neurological causes at 1 year (19 vs 23\%, respectively) despite respective 12-month brain tumor recurrence rates of 46.8 and $76.4 \%$. In a follow-up paper, Aoyama et al., ${ }^{2}$ assessed neurocognitive function in 92 of these patients using the MMSE. They found greater decline in 3-year actuarial MMSE scores in patients receiving WBRT with SRS versus those receiving SRS alone and concluded that the long-term adverse effects of WBRT on neurocognitive function might not be negligible. Soltys et al..$^{22}$ also reported a similar $25 \%$ death rate from neurological causes in 72 patients treated with adjuvant SRS alone following resection, despite a 1-year actuarial distant intracranial failure rate of $53 \%$. In their study using adjuvant SRS/ SRT to the resection bed, Do et al. ${ }^{5}$ also showed that even with a high distant brain recurrence rate of $63 \%$, they were able to achieve prolonged "neurologic-specific survival" and "neurologic deficit-free survival," reporting rates of 75 and $63 \%$, respectively, at 1 year. Forty-seven percent 


\section{Stereotactic radiosurgery boost for oligometastatic brain disease}

of their patients, however, did require salvage WBRT to stabilize their neurological deficits, and the authors concluded that close follow-up with neurological examinations and MR imaging is necessary to detect early recurrence prior to symptoms.

Thirty percent of our patients required salvage WBRT either because of recurrences (in the resection bed or elsewhere in the brain) that were found on surveillance MR imaging or because of failure of salvage SRS for limited disease. No factor examined (patient-, tumor-, or treatment-related) accounted for this, and WBRT was able to be delayed a median of 8.7 months in these patients. Salvage WBRT also played a role in $24-30 \%$ cases reported by Sneed et al. ${ }^{21}$ and Hasegawa et al. ${ }^{7}$ when attempting SRS alone to treat metastatic disease. Aoyama et al. ${ }^{1}$ found that $16.4 \%$ of patients randomized to SRS alone in their study eventually required salvage WBRT.

Our median survival of 15 months is comparable or even superior to results in recently reported series (Table 1) of cases of resected metastatic brain disease. Soltys et al..$^{22}$ and Kresl et al. ${ }^{11}$ also used adjuvant SRS to the operative bed following resection and reported median survivals of 15.1 months and 14.9 months. Do et al. ${ }^{5}$ used either adjuvant SRS or SRT to resection cavities and demonstrated a median survival of 12 months, identical to the result reported by Narayana et al. ${ }^{12}$ in a Phase II study using SRS after resection of solitary metastases. In 2 separate series utilizing adjuvant WBRT following resection of solitary metastases, Patchell and colleagues ${ }^{15,16}$ reported a median survival of 10 and 11 months. Tendulkar et al. ${ }^{23}$ reported their 20-year experience in the surgical treatment of 271 patients with solitary brain metastases (84\% of patients receiving WBRT) and indicated a median survival of 10.2 months, with RTOG RPA Class 1 patients surviving a median of 21.4 months.

The 2 factors associated with improved survival following resection in our work were absence of extracranial disease at presentation and solitary brain metastasis. The RTOG RPA group was not an independent predictor of survival. This is in concordance with the recent communication of Regine et al. ${ }^{19}$ Although some of the older literature had indicated that age ( $>60$ years) had a negative impact on postresection survival, more recent literature has not. ${ }^{14,22}$ We speculate that since age did not impact survival in our population $(\mathrm{p}=0.79)$, the main distinction between RTOG RPA Class 1 and Class 2 (age $>65$ years) became moot. We presume this enhanced performance among the elderly is due to judicious surgical selection, modest postoperative complications, and improved perioperative medical and anesthetic management. Such results would argue against employing any arbitrary age cut-off when deciding on management in an elderly but otherwise healthy patient with a brain metastasis.

\section{Conclusions}

The use of adjuvant SRS to the operative bed following resection of intracranial metastases results in a local intracranial recurrence rate equivalent to that achieved with adjuvant WBRT. Utilizing this approach in combination with SRS for unresected lesions, we were able to spare $70 \%$ of patients the potential acute and chronic morbidity associated with WBRT. Without specific regard to age, this approach can be used in select patients who are healthy, cooperative, and understand that higher rates of recurrence elsewhere in the brain are associated with this approach, requiring surveillance MR imaging of the brain every 3 months.

While we are encouraged by these data, they represent a single-institution retrospective experience. A randomized trial with formal quality-of-life and neurocognitive data would be useful to validate our results.

\section{References}

1. Aoyama H, Shirato H, Tago M, Nakagawa K, Toyoda T, Hatano K, et al: Stereotactic radiosurgery plus whole-brain radiation therapy vs stereotactic radiosurgery alone for treatment of brain metastases: a randomized controlled trial. JAMA 295:2483-2491, 2006

2. Aoyama H, Tago M, Kato N, Toyoda T, Kenjyo M, Hirota S, et al: Neurocognitive function of patients with brain metastasis who received either whole brain radiotherapy plus stereotactic radiosurgery or radiosurgery alone. Int $\mathbf{J}$ Radiat Oncol Biol Phys 68:1388-1395, 2007

3. Chow E, Davis L, Holden L, Tsao M, Danjoux C: Prospective assessment of patient-rated symptoms following whole brain radiotherapy for brain metastases. J Pain Symptom Manage 30:18-23, 2005

4. DeAngelis LM, Delattre JY, Posner JB: Radiation-induced dementia in patients cured of brain metastases. Neurology 39:789-796, 1989

5. Do L, Pezner R, Radany E, Liu A, Staud C, Badie B: Resection followed by stereotactic radiosurgery to resection cavity for intracranial metastases. Int J Radiat Oncol Biol Phys 73:486-491, 2009

6. Gaspar L, Scott C, Rotman M, Asbell S, Phillips T, Wasserman $\mathrm{T}$, et al: Recursive partitioning analysis (RPA) of prognostic factors in three Radiation Therapy Oncology Group (RTOG) brain metastases trials. Int J Radiat Oncol Biol Phys 37:745-751, 1997

7. Hasegawa T, Kondziolka D, Flickinger JC, Germanwala A, Lunsford LD: Brain metastases treated with radiosurgery alone: an alternative to whole brain radiotherapy? Neurosurgery 52:1318-1326, 2003

8. Jagannathan J, Yen CP, Ray DK, Schlesinger D, Oskouian RJ, Pouratian N, et al: Gamma Knife radiosurgery to the surgical cavity following resection of brain metastases. J Neurosurg 111:431-438, 2009

9. Kaplan IW, Meier R: Submucous lipoma of the colon. Am J Gastroenterol 31:673-683, 1959

10. Kondziolka D, Niranjan A, Flickinger JC, Lunsford LD: Radiosurgery with or without whole-brain radiotherapy for brain metastases: the patients' perspective regarding complications. Am J Clin Oncol 28:173-179, 2005

11. Kresl J, Pueschel J, Porter R, Yan E, McDermott M, Sneed P: Surgical resection and postoperative radiosurgery for single brain metastases. Neuro-oncol 5:338, 2003 (Abstract)

12. Narayana A, Chan K, Brennan C, Chang J, Higgins V, Lymberis S, et al: A phase II trial of stereotactic radiosurgery boost following surgical resection for solitary metastases. 2006 ASCO Annual Meeting Proceedings. J Clin Oncol 24 (Suppl):1552, 2006 (Abstract)

13. Nieder C, Schwerdtfeger K, Steudel WI, Schnabel K: Patterns of relapse and late toxicity after resection and whole-brain radiotherapy for solitary brain metastases. Strahlenther Onkol 174:275-278, 1998

14. Noordijk EM, Vecht CJ, Haaxma-Reiche H, Padberg GW, Voormolen JH, Hoekstra FH, et al: The choice of treatment of 
single brain metastasis should be based on extracranial tumor activity and age. Int J Radiat Oncol Biol Phys 29:711-717, 1994

15. Patchell RA, Tibbs PA, Regine WF, Dempsey RJ, Mohiuddin M, Kryscio RJ, et al: Postoperative radiotherapy in the treatment of single metastases to the brain: a randomized trial. JAMA 280:1485-1489, 1998

16. Patchell RA, Tibbs PA, Walsh JW, Dempsey RJ, Maruyama $\mathrm{Y}$, Kryscio RJ, et al: A randomized trial of surgery in the treatment of single metastases to the brain. N Engl J Med 322:494-500, 1990

17. Quigley MR, Fuhrer R, Karlovits S, Karlovits B, Johnson M: Single session stereotactic radiosurgery boost to the post-operative site in lieu of whole brain radiation in metastatic brain disease. J Neurooncol 87:327-332, 2008

18. Regine WF, Huhn JL, Patchell RA, St Clair WH, Strottmann J, Meigooni A, et al: Risk of symptomatic brain tumor recurrence and neurologic deficit after radiosurgery alone in patients with newly diagnosed brain metastases: results and implications. Int J Radiat Oncol Biol Phys 52:333-338, 2002

19. Regine WF, Rogozinska A, Kryscio RJ, Tibbs PA, Young AB, Patchell RA: Recursive partitioning analysis classifications I and II: applicability evaluated in a randomized trial for resected single brain metastases. Am J Clin Oncol 27:505-509, 2004
20. Shaw E, Scott C, Souhami L, Dinapoli R, Kline R, Loeffler J, et al: Single dose radiosurgical treatment of recurrent previously irradiated primary brain tumors and brain metastases: final report of RTOG protocol 90-05. Int J Radiat Oncol Biol Phys 47:291-298, 2000

21. Sneed PK, Suh JH, Goetsch SJ, Sanghavi SN, Chappell R, Buatti JM, et al: A multi-institutional review of radiosurgery alone vs. radiosurgery with whole brain radiotherapy as the initial management of brain metastases. Int J Radiat Oncol Biol Phys 53:519-526, 2002

22. Soltys SG, Adler JR, Lipani JD, Jackson PS, Choi CY, Puataweepong $\mathrm{P}$, et al: Stereotactic radiosurgery of the postoperative resection cavity for brain metastases. Int J Radiat Oncol Biol Phys 70:187-193, 2008

23. Tendulkar RD, Liu SW, Barnett GH, Vogelbaum MA, Toms SA, Jin T, et al: RPA classification has prognostic significance for surgically resected single metastasis. Int J Radiat Oncol Biol Phys 66:810-917, 2006

Manuscript submitted August 14, 2009.

Accepted September 3, 2009.

Address correspondence to: Brian J. Karlovits, D.O., Department of Radiation Oncology, Allegheny General Hospital, 320 East North Avenue, Pittsburgh, Pennsylvania 15212. email: bkarlovi@wpahs. org. 